\title{
Phonon Dispersion Analysis as an Indispensable Tool for Predictions of Solid State Polymorphism and Dynamic Metastability: Case of Compressed Silane
}

\author{
D. KurzydeOWski ${ }^{a, *}$ AND W. Grochala ${ }^{a, b}$ \\ ${ }^{a}$ Faculty of Chemistry, University of Warsaw, Pasteura 1, 02-093 Warsaw, Poland \\ ${ }^{b}$ Interdisciplinary Centre for Mathematical and Computational Modelling, University of Warsaw \\ Pawińskiego 5a, 02-106 Warsaw, Poland \\ (Received September 20, 2010; in final form February 10, 2011)
}

This work is dedicated to Prof. Russell J. Hemley in recognition of his seminal contributions to high pressure sciences

\begin{abstract}
Diamond anvil cell experiments suggest that upon compression above $26.5 \mathrm{GPa}$ silane $\left(\mathrm{SiH}_{4}\right)$ forms a polymeric phase VI, whose crystal structure has not yet been solved. Here we present DFT calculations showing how phonon-guided optimization leads to a polymeric $F d d 2$ structure which is the lowest-enthalpy polymorph of $\mathrm{SiH}_{4}$ above $26.8 \mathrm{GPa}$, and which most probably can be identified as the experimentally observed polymeric phase. The new algorithm of predicting the lowest-energy structures enables simultaneous inspection of the potential energy surface of a given system, calculation of its vibrational properties, and assessment of chances for obtaining a metastable ambient-pressure structure via decompression. Our calculations indicate that at room temperature the differences in the vibrational and entropy terms contributing to the Gibbs free energy of different polymorphs of silane are negligible in comparison with corresponding differences in the zero-point energy corrections, in contrast to earlier suggestions. We also show that the $F d d 2$ polymorph should be metastable upon decompression up to $5 \mathrm{GPa}$, which suggests the possibility of obtaining a polymeric ambient-pressure form of $\mathrm{SiH}_{4}$. Polymeric silane should exhibit facile thermal decomposition with evolution of molecular hydrogen and thus constitute an efficient (12.5 wt\%) material for hydrogen storage.
\end{abstract}

PACS: 81.40.Vw, 63.20.dk, 64.70.K-, 81.30.-t

\section{Introduction}

Silane $\left(\mathrm{SiH}_{4}\right)$ has recently attracted considerable attention as theoretical considerations indicated that this compound might become metallic and superconducting at high compression [1] (similarly to but at lower pressure than the elusive metallic hydrogen $[2,3]$ ). Following this perspective, considerable theoretical and experimental effort has recently been made to understand the influence of high pressure on silane.

Experiments conducted in diamond anvil cells show that $\mathrm{SiH}_{4}$ liquefies at $1.7 \mathrm{GPa}$ and then solidifies at $4 \mathrm{GPa}$ forming phase $\mathrm{III}^{\dagger}$ as evidenced by the Raman spectroscopy [4]. Upon further compression silane enters phase IV (at 6.5 GPa) and then V (at $10 \mathrm{GPa}$ ) [4, 5]. All these phases are molecular and insulating. Phase $\mathrm{V}$, which adopts the $\mathrm{SnBr}_{4}$-type structure $\left(P 2_{1} / c\right.$ symme-

\footnotetext{
* corresponding author; e-mail: dkurzydlowski@chem.uw.edu.pl

$\dagger$ Phases I and II are low temperature polymorphs of $\mathrm{SiH}_{4}$ which are different from the high-pressure ones.
}

try) [5], is stable up to $26.5 \mathrm{GPa}[4,5]$. Above this pressure $\mathrm{SiH}_{4}$ has been reported to undergo a gradual phase transition with phase $\mathrm{V}$ coexisting with a new phase VI over a broad range of pressures. The sluggishness of this phase transformation points to large kinetic barriers which most probably originate from considerable structural difference between phases V and VI. Indeed, vibrational properties of the latter polymorph indicate that it has a polymeric structure [4] although its crystal structure has not been established. This polymorph of silane has also a small band-gap as it is opaque in visible light.

At $50 \mathrm{GPa}$ silane is observed to become amorphous and partially decompose [6]. This new finding contradicts earlier claims that a metallic phase of silane is formed above $50 \mathrm{GPa}[7]$ - the reported "metallic" phase can most probably be attributed to $\mathrm{PtH}_{x}$ (coincidentally obtained from $\mathrm{Pt}$ foil used to measure resistance of the sample, and $\mathrm{H}_{2}$ evolving from silane [6]).

Importantly, silane can be also a very efficient source of $\mathrm{H}_{2}$ fuel (12.5 wt\%). Unfortunately fast release of $\mathrm{H}_{2}$ by thermal decomposition of gaseous $\mathrm{SiH}_{4}$ takes place only at very high temperatures $\left(>350{ }^{\circ} \mathrm{C}\right)[8]$. In this context it would be interesting to verify whether high-pressure treatment of silane would lead to formation of a polymeric form of this compound (by analogy to 
polymeric nitrogen [9]) which could be then quenched to ambient pressure as a metastable (i.e. kinetically stable) polymorph [10]. Such a form of silane could deliver $\mathrm{H}_{2}$ upon decomposition at much lower temperatures than the molecular form. Therefore it seems desirable to computationally verify whether phase VI of $\mathrm{SiH}_{4}$ exhibits a polymeric structure and explore the possibilities of quenching this phase to low pressures.

Previous theoretical studies [11-16] were concentrated on establishing the structure of the claimed metallic form of silane. For this a wide range of strategies of screening the potential energy surface (PES) of this system have been employed: chemical intuition [13], procedure of random sampling [14], molecular dynamics [15] and evolutionary algorithm [16]. In the present study we adopted an alternative strategy for finding the lowest-enthalpy structure of silane. In the first step we calculated the phonon dispersion curves at few selected pressures for a large variety of structures proposed earlier. Whenever the calculations indicated the presence of symmetry-breaking imaginary modes we followed the associated crystal lattice distortions. After geometry re-optimization of the distorted structures we obtained new polymorphs which usually had lower enthalpy than the parent (undistorted) structure. In the final step we recalculated the phonon dispersion curves for the so-obtained novel polymorphs and if imaginary modes were still present, the whole procedure was repeated. This strategy of phonon-guided optimization has been successfully used in the study of solid state polymorphism of $\mathrm{AuF}$ and its adduct with xenon $[17,18]$.

Here we show that this scheme, which combines geometry optimization and phonon calculations, is of enormous value as it comes with four major benefits: (i) it allows for effective screening of the PES of a given solid in a manner that can provide rationalization of the observed phase transitions, (ii) at the same time enables calculation of zero-point energy (ZPE), vibrational and entropy contribution to the Gibbs free energy; (iii) gives information on the vibrational properties of the examined structures, as well as (iv) helps to predict whether a high pressure polymorph could be dynamically stable (metastable) at a given pressure.

Applying this scheme in the case of compressed silane we show in this work that: (i) starting from a structure which is thermodynamically uncompetitive in the examined range of pressures (below $50 \mathrm{GPa}$ ) we are able to obtain the theoretical ground state structure of silane at these conditions; (ii) we show also that differences in the vibrational and entropy contributions to the Gibbs free energy calculated for different solid-state polymorphs of $\mathrm{SiH}_{4}$ largely cancel out; (iii) most importantly, our calculations indicate that the $F d d 2$ structure should be metastable at pressures close to ambient conditions which suggests that polymeric silane obtained at high pressure might be quenchable.

\section{Results and discussion}

\subsection{Phonon-guided optimization}

Although a multitude of various polymorphs of silane have been suggested in the previous works, yet the issue of the dynamic stability has not been addressed for most of them. This is, we suppose, mainly due to substantial computational burden related to time-consuming calculations of phonon dispersion for every crystal structure at every pressure point.

Initially we verified the phonon stability for a broad spectrum of the earlier reported structures [5, 7, 13-15]. The calculations show that the seemingly simple $\mathrm{SiH}_{4}$ system has a very complicated PES as all of the examined structures exhibit phonon instabilities for at least one pressure point from the range 15-125 GPa [19]. Surprisingly the $P 2_{1} / c$ structure of phase $\mathrm{V}$ does not exhibit imaginary modes up to $91 \mathrm{GPa}$ - see Electronic Supplementary Information (ESI).

Among others we have investigated the $I-42 d$ polymorph which has been previously briefly discussed in Refs. [14] and [16]. We find that this structure is close to being the ground state of the $\mathrm{SiH}_{4}$ system at $50 \mathrm{GPa}$ (see ESI), but is dynamically unstable at $15 \mathrm{GPa}$ with two imaginary vibrations at $\Gamma$-point (one of $B 1$ symmetry and $\tilde{\nu}=143 \mathrm{~cm}_{(i)}^{-1}$, second of $B 2$ symmetry and $\left.\tilde{\nu}=945 \mathrm{~cm}_{(i)}^{-1}\right)$. The larger absolute value of the imaginary frequency points to a steeper slope in the PES and consequently following the "most imaginary" mode gives the best chance for obtaining the lowest lying minimum of the PES. Thus, we choose to follow the distortion introduced by the $B 2$ mode.

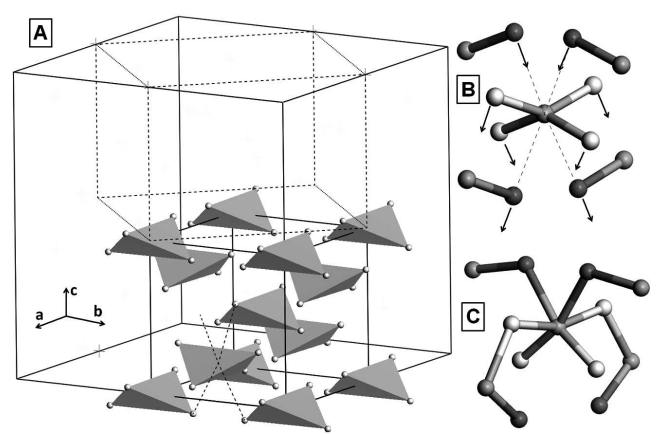

Fig. 1. (A) The crystal structure of $I-42 d$ at $15 \mathrm{GPa}$ (small black box represents the unit cell, the large one the $2 \times 2 \times 2$ supercell used for calculating the phonon dispersion curves). Tetrahedra formed by four $\mathrm{Si}-\mathrm{H}$ bonds are marked in grey, dashed lines mark additional H. .Si contacts. The unit cell of the structure obtained after following the imaginary $B 2$ mode is marked with dashed lines. (B) Movement of atoms in the B2 vibration (grey balls - Si; white balls - H from $1^{\text {st }}$ coordination sphere of $\mathrm{Si}$, dark grey balls - $\mathrm{H}$ from neighbouring $\mathrm{SiH}_{4}$ tetrahedra). (C) View of the corner-linked distorted $\mathrm{SiH}_{6}$ octahedron present in the Fdd2 structure. 
The structure of $I-42 d$ at $15 \mathrm{GPa}$ is composed of distorted $\mathrm{SiH}_{4}$ tetrahedra ( $\mathrm{Si}-\mathrm{H}$ bonds at $1.536 \AA, \mathrm{H}-\mathrm{Si}-\mathrm{H}$ angle equal to $143^{\circ}$ ) with all four hydrogen atoms forming additional $\mathrm{H}$. . Si contacts to neighbouring $\mathrm{SiH}_{4}$ units (at $1.926 \AA$ ) - see Fig. 1. The B2 mode consists mainly of the motion of the hydrogen atoms - they move along the axis given by the H. . Si contact as depicted in Fig. 1. These movements result in substantial alternation of these four contacts splitting them into two short ones $(1.658 \AA)$ and two longer ones $(2.294 \AA)$. The distortion introduced by the $B 2$ vibration leads to a structure with $\boldsymbol{a}^{\prime}, \boldsymbol{b}^{\prime}$, and $\boldsymbol{c}^{\prime}$ cell vectors equal to $\boldsymbol{a}+\boldsymbol{b}, \boldsymbol{a}+\boldsymbol{b}$, and $\boldsymbol{c}$, respectively (where $\boldsymbol{a}, \boldsymbol{b}, \boldsymbol{c}$ are cell vectors of $I-42 d$ ). The considerable shortening of two of the four H. . Si contacts in the $B 2$ imaginary mode points to a tendency of the system to form a polymeric structure. Indeed, after subsequent geometry re-optimization of the distorted structure we arrive at a structure of $F d d 2$ symmetry (this structure has been previously reported in Refs. [11] and [12]) which exhibits a $2+2+2$ coordination with two short (terminal) $\mathrm{Si}-\mathrm{H}$ bonds and four longer (bridging) ones. Thus the coordination of $\mathrm{Si}$ by $\mathrm{H}$ is in a form of a distorted octahedron with every octahedron linked to another four by four of its corners. In fact this structure type is the same as adopted by some $\mathrm{MF}_{4}$ compounds
$(\mathrm{M}=\mathrm{Ir}, \mathrm{Pd}, \mathrm{Pt})$ [20].

The transition from the $I-42 d$ to the $F d d 2$ polymorph is favoured in enthalpy by $0.31 \mathrm{eV} /$ f.u. at $15 \mathrm{GPa}$ which shows how effective following of the imaginary modes can be when searching for the global energy minimum for a given system. We notice that due to the complicated nature of any PES it is not required that the parent (dynamically unstable) structure is close in energy to the global minimum of the system. Following imaginary modes of very uncompetitive (high-enthalpy) structure often enables much faster identification of the global minimum of the PES than distorting a polymorph which has a lower enthalpy.

In the case of $I$ - $42 d$ to $F d d 2$ transition a $\Gamma$-point mode has been followed and consequently the symmetry operators of the latter polymorph form a sub-group of $I-42 d$. Generally this does not have to be the case as imaginary modes from the whole Brillouin zone can be followed. These will often result in distorted structures of very low symmetry which after geometry re-optimization can change severely (due to small number of symmetry restrictions). Thus our method of phonon-guided optimization in principle does not have to lead to structures which are related by symmetry with the parent structure.

TABLE

Cell vectors (in $\AA$ and ${ }^{\circ}$ ), volume per $\mathrm{SiH}_{4}$ unit (in $\AA^{3}$ ) and atomic fractional coordinates for $P 2_{1} / c$ and $F d d 2$ polymorphs at selected pressures (in GPa). For volume vs. pressure plot see ESI.

\begin{tabular}{|c|c|c|c|c|c|c|c|c|}
\hline Phase & $p$ & $a$ & $b$ & $c$ & $\beta$ & $V / Z$ & $\mathrm{Si}$ & $\mathrm{H}$ \\
\hline \multirow{7}{*}{$P 2_{1} / c$} & \multirow{5}{*}{5} & \multirow{4}{*}{6.481} & \multirow{4}{*}{4.302} & \multirow{4}{*}{6.515} & \multirow{4}{*}{104.5} & \multirow{4}{*}{43.96} & \multirow{4}{*}{$(0.249,0.573,0.360)$} & H1: $(0.060,0.565,0.177)$ \\
\hline & & & & & & & & $\mathrm{H} 2:(0.313,0.900,0.417)$ \\
\hline & & & & & & & & H3: $(0.427,0.408,0.302)$ \\
\hline & & & & & & & & $\mathrm{H} 4:(0.197,0.417,0.544)$ \\
\hline & & \multirow{3}{*}{5.880} & \multirow{3}{*}{3.955} & \multirow{3}{*}{5.912} & \multirow{3}{*}{104.3} & \multirow{3}{*}{33.31} & \multirow{3}{*}{$(0.249,0.578,0.367)$} & $\mathrm{H} 1:(0.043,0.570,0.165)$ \\
\hline & \multirow{2}{*}{15} & & & & & & & $\mathrm{H} 2:(0.321,0.930,0.430)$ \\
\hline & & & & & & & & $\begin{array}{l}\text { H3: }(0.444,0.400,0.300) \\
\text { H4: }(0.190,0.402,0.565)\end{array}$ \\
\hline \multirow{6}{*}{$F d d 2$} & \multirow{2}{*}{30} & \multirow{2}{*}{6.924} & \multirow{2}{*}{7.057} & \multirow{2}{*}{4.237} & \multirow{2}{*}{90} & \multirow{2}{*}{25.88} & \multirow{2}{*}{$(0.5,0.5,0.991)$} & H1: $(0.597,0.411,0.667)$ \\
\hline & & & & & & & & $\mathrm{H} 2:(0.374,0.364,0.449)$ \\
\hline & \multirow{2}{*}{40} & \multirow{2}{*}{6.607} & \multirow{2}{*}{7.106} & \multirow{2}{*}{4.089} & \multirow{2}{*}{90} & \multirow{2}{*}{24.00} & \multirow{2}{*}{$(0.5,0.5,0.992)$} & $\mathrm{H} 1:(0.612,0.424,0.660)$ \\
\hline & & & & & & & & H2: $(0.393,0.350,0.455)$ \\
\hline & \multirow{2}{*}{50} & \multirow{2}{*}{6.492} & \multirow{2}{*}{6.985} & \multirow{2}{*}{3.998} & \multirow{2}{*}{90} & \multirow{2}{*}{22.66} & \multirow{2}{*}{$(0.5,0.5,0.994)$} & H1: $(0.612,0.426,0.656)$ \\
\hline & & & & & & & & H2: $(0.396,0.351,0.458)$ \\
\hline
\end{tabular}

According to our calculations the Fdd2 polymorph becomes the ground state structure of compressed silane at 25.6 GPa (26.8 GPa when ZPE corrections are taken into account) as can be seen in Fig. 2. This value is in a very good agreement with the experimentally observed transition pressure of $26.5 \mathrm{GPa}$ (for the pressure evolution of structural parameters of P21/c and Fdd2 see Table). We must note that the Fdd2 structure has already been proposed by Martinez-Canales et al. as a plausible candidate for phase VI of $\mathrm{SiH}_{4}$ between $25 \mathrm{GPa}$ and $55 \mathrm{GPa}[12]$. Our findings corroborate this suggestion that the molecular $P 2_{1} / c$ polymorph should trans- form to polymeric $F d d 2$ at $25 \mathrm{GPa}^{\ddagger}$. The use of phonon-guided optimization enables us to further propose that

\footnotetext{
$\ddagger$ The X-ray diffraction pattern of $F d d 2$ does not reproduce two new peaks observed for compressed $\mathrm{SiH}_{4}$ above $25 \mathrm{GPa}$ (Ref. [6]). We notice that these two peaks cannot be unambiguously assigned to a new phase of $\mathrm{SiH}_{4}$ as Ref. [7] suggests that the $P 2_{1} / c$ polymorph is stable up to $50 \mathrm{GPa}$. It seems that because of considerable kinetic barriers observation of phase VI of silane strongly depends on the temperature at which the experiment was conducted.
} 
the bending vibration of the $\mathrm{SiH}_{4}$ tetrahedra could possibly offer a path along which this transition proceeds.

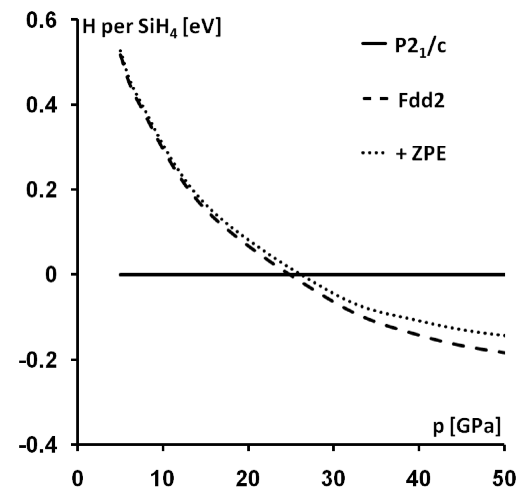

Fig. 2. Enthalpy per one $\mathrm{SiH}_{4}$ unit vs. pressure for the $P 2_{1} / c$ (solid line) and $F d d 2$ (dashed line) polymorphs. The dotted line shows the relative enthalpies of the two structures after applying the ZPE correction. The enthalpy and the ZPE corrections are referenced at each pressure point to that of $P 2_{1} / c$.

\subsection{ZPE, vibrational and entropy terms to the Gibbs free energy}

Inclusion of the ZPE term can have a major impact on the free energy of synthesis reactions for many hydrides and deuterides, as shown in theoretical studies for $\mathrm{LiMH}_{4}$ systems $(\mathrm{M}=\mathrm{Al}, \mathrm{B})$ [21]. This is mostly due to substantial ZPE of the $\mathrm{H}_{2}$ molecule, which is one of the substrates of the synthesis reactions. However, the impact of the ZPE term on relative stability of various polymorphs of extended hydrides, has not been meticulously studied to date. We examined the ZPE term as well as the magnitude of other two terms contributing to the Gibbs free energy at non-zero temperatures, namely the vibrational energy $\left(E_{\mathrm{vib}}\right)$ and the entropy term $(S T)$ for various polymorphs of silane. Our calculations show that for a broad range of pressures the differences in the vibrational $\left(\Delta E_{\mathrm{vib}}\right)$ and entropy $(-\Delta S T)$ terms between different structures are of the order of $10 \mathrm{meV}$ at room temperature and thus they are small compared to the corresponding differences in $E_{\mathrm{ZPE}}$ (up to $100 \mathrm{meV}$ ) for details see ESI. Moreover, $\Delta E_{\mathrm{vib}}$ and $-\Delta S T$ in most cases have opposite signs and thus they cancel out. This shows that neither of these two terms should have a major influence on the thermodynamic stability of different phases of silane (at least at room temperature or below), in contrast to earlier suggestions [7].

\subsection{Dynamic properties}

As already mentioned, Fdd2 is polymeric with the Si atoms exhibiting a $2+2+2$ coordination with two terminal $\mathrm{Si}-\mathrm{H}$ bonds of $1.47 \AA$ at $30 \mathrm{GPa}$ and four bridging ones at $1.59 \AA$ and $1.65 \AA$ at $30 \mathrm{GPa}$. In contrast, at the same pressure the $P 2_{1} / c$ structure still exhibits isolated $\mathrm{SiH}_{4}$ units with an average $\mathrm{Si}-\mathrm{H}$ bond length of $1.47 \AA$
(1.2\% contraction with respect to the silane molecule at ambient pressure) indicating that at this compression $P 2_{1} / c$ is still a molecular structure (the shortest intermolecular contact is $2.10 \AA$ ). This is confirmed by the analysis of the phonon structure of this polymorph (Fig. 3). The $\mathrm{Si}-\mathrm{H}$ stretching modes $\left(2150 \mathrm{~cm}^{-1}\right.$ to $2300 \mathrm{~cm}^{-1}$ ) have small dispersion and their frequencies are well separated from those corresponding to the $\mathrm{H}-\mathrm{Si}-\mathrm{H}$ bending modes $\left(900 \mathrm{~cm}^{-1}\right.$ to $1080 \mathrm{~cm}^{-1}$ at $\Gamma$ -point).

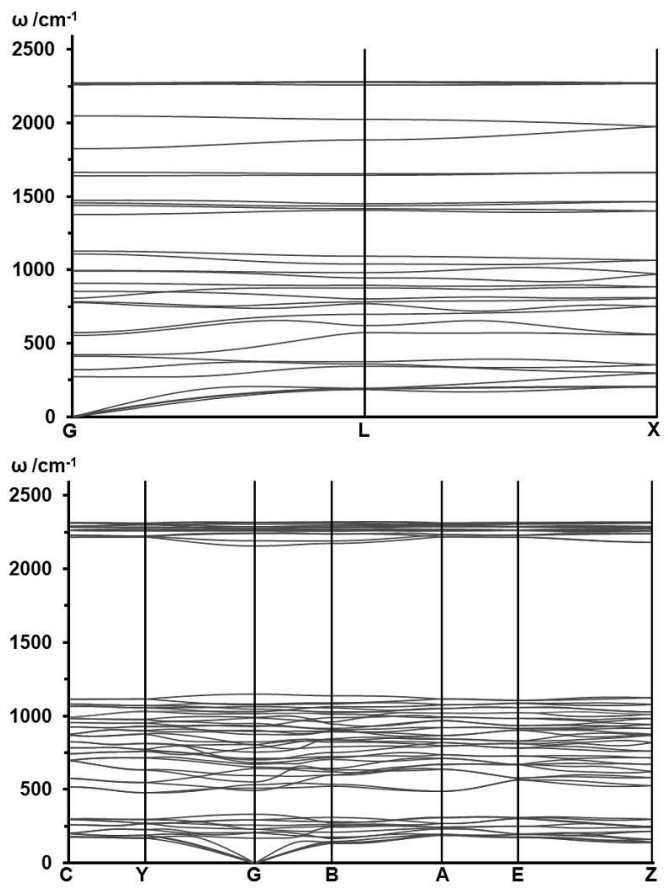

Fig. 3. The phonon dispersion relations for polymorphic $F d d 2$ (top) and molecular $P 2_{1} / c$ (bottom) at $30 \mathrm{GPa}$.

The Fdd2 structure retains its polymeric framework upon decompression up to $5 \mathrm{GPa}$ (the lowest pressure considered by us) [22]. This is evidenced by the phonon dispersion curves calculated at each pressure for this structure. Apart from the stretching and bending vibrations of the terminal hydrogen atoms (with frequencies similar to those calculated for $P 2_{1} / c$ ) the $F d d 2$ polytype exhibits modes in the $1400-2050 \mathrm{~cm}^{-1}$ region $(\Gamma$-point at $30 \mathrm{GPa}$ ) which are absent for $P 2_{1} / c$. These can be assigned to the stretching $\left(2050 \mathrm{~cm}^{-1}\right.$ to $\left.1660 \mathrm{~cm}^{-1}\right)$ and bending $\left(1500 \mathrm{~cm}^{-1}\right.$ to $\left.1400 \mathrm{~cm}^{-1}\right)$ of the $\mathrm{Si}-\mathrm{H}-\mathrm{Si}$ polymeric bridges. Softening of the stretching modes and stiffening of the bending modes, as compared to molecular $P 2_{1} / c$ phase, is a clear signature of polymeric nature of the $F d d 2$ phase.

\subsection{Metastability}

Calculations of the phonon dispersion curves indicate that the $F d d 2$ structure should be metastable upon decompression as it does not exhibit imaginary modes down 
to $5 \mathrm{GPa}$. This pressure is the lowest considered in our study. Geometry optimizations of molecular compounds conducted at lower compressions are subject to large errors due to the insufficient description of the van der Waals interactions by density functional theory (DFT) methods. Nevertheless, our results indicate that upon low-temperature decompression the $F d d 2$ structure might be retained at ambient conditions. At $5 \mathrm{GPa}$ the enthalpy difference between the $P 2_{1} / c$ and $F d d 2$ polymorphs is over $0.5 \mathrm{eV}$ per f.u. (Fig. 2). Therefore, the $F d d 2$ structure at low pressure would represent a severely destabilized form of silane, which should thermally decompose at much milder temperature conditions than any molecular polymorph of $\mathrm{SiH}_{4}$.

\section{Conclusions}

In this work we presented a phonon-based scheme for finding the lowest-enthalpy structure of a given solid state system. Applying it in the case of compressed silane showed that majority of different high pressure polymorphs of this compound reported in the literature are not dynamically stable. Following imaginary modes exhibited by unstable structure leads to quick localization of the minimum of the potential energy surface of a given system as exemplified by the case of the $F d d 2$. Our calculations corroborate the suggestion that the semi-molecular $F d d 2$ polymorph constitutes phase VI of silane (suggested to be stable above $26 \mathrm{GPa}$ ). We examine the vibrational properties of this phase at high pressure and compare them to those exhibited by the molecular $P 2_{1} / c$ form. It turns out that vibrational and entropy contributions to free enthalpy largely cancel out, in contrast to previous suggestions. Finally, calculations of phonon dispersion relations for $F d d 2$ show that this structure is metastable upon decompression up to at least $5 \mathrm{GPa}$ and thus, if quenched, it might constitute a novel thermodynamically-destabilized H-rich material for hydrogen storage in the solid state.

\section{Methods}

Our approach is based on the density functional theory - a current method of choice for calculating structure and properties of crystals. Our calculations utilized the Perdew-Burke-Ernzerhof exchange-correlation functional [23]. The projector-augmented wave (PAW) method [24] was used as implemented in the VASP code [25].

The direct method, as implemented in PHONON [26], was used for calculating phonon dispersion curves and phonon density of states as well as zero-point vibration, vibrational and entropy terms. The supercells consisted of 160 and 80 atoms for $P 2_{1} / c$ and $F d d 2$ structures, respectively. Supercells of similar size were used for calculations presented in the ESI. Energy calculations for the supercells generated by PHONON were done using VASP.
For geometry optimizations and phonon calculations the cut-off energy of the plane waves was set to $600 \mathrm{eV}$ with a self-consistent field (SCF) convergence criterion of $1 \times 10^{-5} \mathrm{eV}$. The $k$-point mesh was generated via the Monkhorst-Pack scheme [27] for every structure in such a way that the spacing between the $k$-points was approximately $0.3 \AA^{-1}$.

Comparison of wave numbers of the calculated and experimentally observed Raman bands, is given in the ESI.

\section{Acknowledgments}

This research was supported from the funds of Polish Ministry of Science and Higher Education under contract No. 37/6PR UE/2007/7, as well as by the Foundation for Polish Science "TEAM" Programme co-financed by the EU European Regional Development Fund. Discussions with Dr. Mariana Derzsi are gratefully acknowledged.

\section{Note added in proof}

When this manuscript was in proof a new report on the synthesis of $\mathrm{SiH}_{4}$ at high pressure has appeared see Ref. [28].

\section{References}

[1] N.W. Ashcroft, Phys. Rev. Lett. 92, 187002 (2004).

[2] C. Narayana, H. Luo, J. Orloff, A.L. Ruoff, Nature (London) 393, 46 (1998).

[3] B. Edwards, N.W. Ashcroft, Nature (London) 388, 652 (1997).

[4] X.J. Chen, V.V. Struzhkin, Y. Song, A.F. Goncharov, M. Ahart, Z. Liu, H.K. Mao, R.J. Hemley, Proc. Natl. Acad. Sci. USA 105, 20 (2008).

[5] O. Degtyareva, M. Martinez-Canales, A. Bergara, X.J. Chen, Y. Song, V.V. Struzhkin, H.K. Mao, R.J. Hemley, Phys. Rev. B 76, 064123 (2007).

[6] O. Degtyareva, J.E. Proctor, C.L. Guillaume, E. Gregoryanz, M. Hanfland, Solid State Commun. $\mathbf{1 4 9}$ 1583 (2009).

[7] M.I. Eremets, I.A. Trojan, S.A. Medvedev, J.S. Tse, Y. Yao, Science 319, 1506 (2008).

[8] W. Grochala, P.P. Edwards, Chem. Rev. 104, 1283 (2004).

[9] M.I. Eremets, A.G. Gavriliuk, I.A. Trojan, D.A. Dzivenko, R. Boehler, Nat. Mat. 3, 558 (2004).

[10] For a commentary on metastability at high pressure see: V.V. Brazhkin, A.G. Lyapin, Nat. Mat. 3, 497 (2004).

[11] X.J. Chen, J.L. Wang, V.V. Struzhkin, H.K. Mao, R.J. Hemley, H.Q. Lin, Phys. Rev. Lett. 101, 077002 (2008).

[12] M. Martinez-Canales, A.R. Oganov, Y. Ma, Y. Yan, A.O. Lyakhov, A. Bergara, Phys. Rev. Lett. 102, 087005 (2009).

[13] J. Feng, W. Grochala, T. Jaroń, R. Hoffmann, A. Bergara, N.W. Ashcroft, Phys. Rev. Lett. 96, 017006 (2006). 
[14] C.J. Pickard, R.J. Needs, Phys. Rev. Lett. 97, 045504 (2006).

[15] Y. Yao, J.S. Tse, Y. Ma, K. Tanaka, Europhys. Lett. 78, 37003 (2007)

[16] D.Y. Kim, R.H. Scheicher, S. Lebègue, J. Prasongkit, B. Arnaud, M. Alouani, R. Ahuja, Proc. Natl. Acad. Sci. USA 105, 16454 (2008).

[17] D. Kurzydłowski, W. Grochala, Chem. Commun., 1073 (2008)

[18] D. Kurzydłowski, W. Grochala, Z. Anorg. Allg. Chem. 634, 1082 (2008).

[19] For details concerning the dynamic stability of different high pressure polymorphs of $\mathrm{SiH}_{4}$ see Electronic Supplementary Information (ESI), available at http://tnfm.icm.edu.pl//images/files/ sih4_hp_esi_.pdf.

[20] W.J. Casteel Jr., D.H. Lohmann, N. Bartlett, J. Flourine Chem. 112, 165 (2001).

[21] T.J. Frankcombe, G.J. Kroes, A. Züttel, Chem. Phys. Lett. 405, 73 (2005); T.J. Frankcombe, G.J. Kroes, Chem. Phys. Lett. 423, 102 (2006).
[22] For the pressure evolution of bond lengths see ESI.

[23] J.P. Perdew, K. Burke, M. Ernzerhof, Phys. Rev. Lett. 77, 3865 (1997).

[24] P.E. Blöchl, Phys. Rev. B 50, 17953 (1994).

[25] G. Kresse, J. Futhmüller, Phys. Rev. B 54, 11169 (1996); G. Kresse, J. Futhmüller, Comput. Mater. Sci. 6, 15 (1996); G. Kresse, D. Joubert, Phys. Rev. B59, 1758 (1999).

[26] K. Parliński, Z.Q. Li, Y. Kawazoe, Phys. Rev. Lett. 78, 4063 (1997).

[27] H.J. Monkhorst, J.D. Pack, Phys. Rev. B 13, 5188 (1976).

[28] M. Hanfland, J.E. Proctor, C.L. Guillaume, O. Degtyareva, E. Gregoryanz, Phys. Rev. Lett. 106, 095503 (2011). 